Report 99-022

Tandem fluid queues fed by homogeneous on-off sources Samuli Aalto and Werner Scheinhardt

ISSN: 1389-2355 


\title{
TANDEM FLUID QUEUES FED BY HOMOGENEOUS ON-OFF SOURCES
}

\author{
SAMULI AALTO AND WERNER SCHEINHARDT
}

\begin{abstract}
We consider a tandem fluid model with multiple consecutive buffers. The input of buffer $j+1$ is the output from buffer $j$, while the first buffer is fed by a, possibly infinite, number of independent homogeneous on-off sources. The sources have exponentially distributed silent periods and generally distributed active periods. Under the assumption that the input rate of one source is larger than the maximum output rate of the first buffer, we are able to characterize the output from each buffer. Due to this fact we find (i) an equation for the Laplace-Stieltjes transform of the marginal content distribution of any buffer $j \geq 2$, (ii) explicit expressions for corresponding moments, and (iii) an explicit expression for the correlation between two buffer contents, again from the second buffer on. These results make use of a key observation concerning the aggregate contents of several consecutive buffers. For the case in which the active periods of the sources are exponential, the Laplace-Stieltjes transform is inverted. If there is only one source, all results are also valid for the first buffer.
\end{abstract}

\section{INTRODUCTION}

In the area of modern telecommunication systems, fluid queues are often used as burst scale models for multiplexers, see e.g. [16]. In such models, a fluid queue (or fluid buffer) receives input at a rate which is determined by some stochastic process $X(t)$. Often this process is integer-valued and the input rate is $i c_{0}$ at times when $X(t)=i$. In addition, the buffer leaks fluid at a fixed rate $c_{1}$ as long as it is not empty. The physical interpretation of such models is as follows. A multiplexer consists of a number of input lines, a buffer and an output line. The parameters $c_{0}$ and $c_{1}$ can be thought to be the transmission rates of each input line and of the output line, respectively. At time $t$, there are $X(t) \geq 0$ sources active, each of which is generating fluid (information), feeding this into the buffer at a constant rate $c_{0}$. Whenever the total input rate exceeds the capacity of the output line, fluid is temporarily stored in the buffer. As long as there is fluid in the buffer, the output line transmits at full capacity $c_{1}$.

The sources are often assumed to be on-off sources. When active, such a source generates fluid (at rate $c_{0}$ ), and when silent, no fluid is generated. The consecutive active and silent periods of each source constitute an alternating renewal process. Moreover, the sources are often assumed to be homogeneous and independent. In that case, the number of active sources $X(t)$ behaves like the number of customers in a certain queue. Assuming that the silent periods of the sources are exponential and the active periods have a general distribution, this queue is either a so-called $M / G / N / N / N$ queue if the number of sources, $N$, is finite, or it is an $M / G / \infty$ queue if

Date: June 29, 1999.

1991 Mathematics Subject Classification. 60K25.

Key words and phrases. Tandem fluid queue, buffer content, output process, on-off source. 
$N=\infty$. Classical examples of these models can be found in [3] and [14], respectively. As an aside, we note that in some papers the fluid queue driven by an $M / G / \infty$ queue is called an $M / G / 1$ gradual input queue, see e.g. $[6,13]$.

Although many papers consider these types of fluid queues (see e.g. the survey paper [4] and the references therein), there are hardly any explicit results concerning the buffer content distribution when the on times of the sources have a general distribution. For example, for the fluid queue driven by an $M / G / \infty$ queue the mean buffer content seems to be known only when $c_{0}=c_{1}$ ( (ee $[19,13]$ ).

Besides the buffer content, it is important to characterize the output process, one reason being that this knowledge enables the analysis of a tandem system. Some results have been obtained in this area, one important result being that whenever $c_{0} \geq c_{1}$, the output process looks like another on-off source with exponential silent periods and generally distributed active periods. Rubinovitch [17] and Cohen [6] found that if $c_{0}=c_{1}$, then the active periods on the output line are distributed like busy periods in a certain $M / G / 1$ queue. Recent studies [5,2] show that similar results are valid also in the case $c_{0}>c_{1}$. However, when $c_{0}<c_{1}$, the output process is much more complicated. In this case the output has been characterized only for the model with exponential sources, see [1].

In this paper we use these results to consider a tandem fluid queue fed by a finite or an infinite number of independent homogeneous on-off sources with exponential silent periods and generally distributed active periods. Thus, the input rate to the first buffer is modulated by the appropriate queue length process $X(t)$, and the output from buffer $j$ is the input to buffer $j+1$. In view of the previous paragraph it is no surprise that we assume $c_{0} \geq c_{1}$, leaving the opposite case for future research. As an aside, we remark that this tandem fluid queue is essentially different from ordinary tandem queues, where each workload process has jumps. Moreover the sizes of these jumps are all random, whereas in our model all randomness relates to the behaviour of the sources, the network itself being deterministic.

Kella and Whitt [9] considered a tandem fluid model where the flow between consecutive buffers is deterministic (as in our model) but the input flow to the first buffer is instantaneous. They assumed that the input process is a non-decreasing Lévy process. Their main results concern the case where the input process is a compound Poisson process. This can be seen as a limiting case of our model with a single source $(N=1)$ : let the rate $c_{0}$ tend to $\infty$ and the active periods tend to 0 in such a way that the burst sizes (i.e. the product of $c_{0}$ and the length of an active period) converge to a proper random variable. In some more recent papers, see e.g. [10,11], Kella considers more general tandem fluid networks, also with instantaneous input and hence different from ours. In [12], some Markov-modulated fluid networks are considered. For a particular model in this setting, namely a two-node tandem fluid queue driven by one on-off source with exponential on times and off times, the joint content distribution was found explicitly in $[15,18]$.

Our main results in this paper are as follows. We show that the output from each buffer in the tandem looks like an on-off source with exponential off times and on times distributed as busy periods in an $M / G / 1$ queue. Due to this fact we find (i) an implicit equation for the Laplace-Stieltjes transform of the marginal content distribution of any buffer $j \geq 2$, (ii) explicit expressions for corresponding moments, and (iii) an explicit expression for the correlation between two buffer contents, again 
from the second buffer on. For the case in which the active periods of the sources are exponential, the Laplace-Stieltjes transform is inverted, as in [18]. If there is only one source, all results are also valid for the first buffer.

The paper is organized as follows. First, in Section 2, we introduce the tandem fluid queue and make some key observations that hold under rather general conditions. The most important observation gives a relation between the behaviour of the aggregate contents of several consecutive buffers and the behaviour of the content of a single buffer in a related tandem system. In Section 3, we present some (known) results concerning the model with a single source and a single buffer. These results serve as a basis for the main results which are presented in Section 4, where we consider the tandem fluid queues driven by the $M / G / N / N / N$ and $M / G / \infty$ queues.

\section{KEY OBSERVATIONS}

Consider a tandem fluid queue driven by a stochastic process $X(t)$. In later sections we will further specify this process, but since the observations we make in this section hold in a more general sense, we will here only assume that $X(t)$ takes values in $\{0,1, \ldots, N\}$ for some $N>0$ (possibly $N=\infty$ ). Assume that there are $M$ fluid buffers connected in series. Let $c_{j}$ denote the leak rate (i.e. the maximum output rate) of the $j$ th buffer. The output from buffer $j$ is the input to buffer $j+1$. The input rate to the first buffer is $X(t) c_{0}$.

We denote the content of buffer $j$ at time $t$ by $Z_{j}(t)$. We assume that the system is stable, and let $Z_{j}$ be distributed according to the limiting distribution of $Z_{j}(t)$ as $t \rightarrow \infty$. Since we are interested in this limiting behaviour, we may assume that $Z_{j}(0)=0$ for all $j$, and we will henceforth do so.

We assume, without loss of generality, that

$$
N c_{0}>c_{1}>c_{2}>\ldots>c_{M} \text {. }
$$

When this condition does not hold, one or more buffers will always be empty in stationarity, so that these buffers can be removed from the tandem (ensuring validity of (2.1) in the modified system) before employing the analysis in this paper. We can now make the following observation.

Proposition 2.1. Consider the tandem fluid queue model described above. Let $i<$ $j \leq M$. Then, for all $t \geq 0$,

$$
Z_{i}(t)>0 \Rightarrow Z_{j}(t)>0
$$

Proof. Suppose $Z_{i}(t)>0$ for some $i<M$ and $t>0$. Define $t_{0}=\sup \left\{s<t \mid Z_{i}(s)=\right.$ $0\}$. Then buffer $i+1$ has experienced an inflow at rate $c_{i}$ during the interval $\left(t_{0}, t\right]$. Since $c_{i}>c_{i+1}$, this has resulted in an increase of the fluid level in buffer $i+1$, so that $Z_{i+1}(t)>0$. The proposition now follows by incluction.

Finally, if $N>1$, we assume that

$$
c_{0} \geq c_{1}
$$

This is a rather restrictive assumption, but it guarantees that the output from the first buffer looks like another on-off source. 
Under Assumptions (2.1) and (2.2), it now follows formally that the evolutions of the processes $Z_{j}(t)$ are given by

$$
\begin{aligned}
& Z_{1}(t)=\int_{0}^{t}\left(c_{0} X(u)-c_{1}\right) 1\left\{Z_{1}(u)>0\right\} d u \\
& Z_{j}(t)=\int_{0}^{t}\left(c_{j-1} \mathbf{1}\left\{Z_{j-1}(u)>0\right\}-c_{j} \mathbf{1}\left\{Z_{j}(u)>0\right\}\right) d u, \quad j \geq 2,
\end{aligned}
$$

where $1\{A\}$ is the indicator function of the event $\{A\}$. We now present another observation, characterizing the distribution of a sum of the contents of consecutive buffers.

Proposition 2.2. Consider the tandem fluid queue model described above. Let $j \geq$ $i \geq 1$. Then

$$
Z_{i}+Z_{i+1}+\ldots+Z_{j} \sim \tilde{Z}_{i}
$$

where $\tilde{Z}_{i}$ refers to the stationary version of the content of buffer $i$ in a modified tandem fluid queue model with $i$ buffers, where the input rate into the first buffer is modulated by $X(t)$ in the same way as before, but the leak rates of the buffers are $c_{1}, c_{2}, \ldots, c_{i-1}, c_{j}$.

Proof. We couple the original and the modified tandem system such that they are regulated by the same process $X(t)$. Assume first that $i \geq 2$. For the modified system, we find that $\tilde{Z}_{i}(t)$ satisfies

$$
\tilde{Z}_{i}(t)=\int_{0}^{t}\left(c_{i-1} 1\left\{Z_{i-1}(u)>0\right\}-c_{j} 1\left\{\tilde{Z}_{i}(u)>0\right\}\right) d u .
$$

In the original system, we find, by summing (2.4) for $k=i, \ldots, j$, that

$$
Z_{i j}(t) \equiv Z_{i}(t)+Z_{i+1}(t)+\ldots+Z_{j}(t)
$$

satisfies

$$
Z_{i j}(t)=\int_{0}^{t}\left(c_{i-1} 1\left\{Z_{i-1}(u)>0\right\}-c_{j} 1\left\{Z_{j}(u)>0\right\}\right) d u .
$$

By applying Proposition 2.1, we can see that $Z_{i j}(u)>0$ (that is, $Z_{k}(u)>0$ for some $k=i, \ldots, j$,$) implies Z_{j}(u)>0$, while clearly also the converse is true. Therefore we can replace $\mathbf{1}\left\{Z_{j}(u)>0\right\}$ by $1\left\{Z_{i j}(u)>0\right\}$ in (2.7). It follows that $\tilde{Z}_{i}(t)=Z_{i j}(t)$ for all $t \geq 0$. A similar reasoning holds when $i=1$, replacing $c_{i-1} \mathbf{1}\left\{Z_{i-1}(u)>0\right\}$ by $c_{0} X(u)$ in both (2.5) and (2.7). Hence the proposition follows.

Notice in particular that, by taking $i=1$, we find that $Z_{1}+\ldots+Z_{j}$ is distributed as the content of a fluid buffer with leak rate $c_{j}$ and driven by $X(t)$.

\section{PRELiminaries: SiNGle SOURCE, Single BUfFER}

In this section we present some known results concerning the characterization of the output process and the buffer content distribution in a system with only one source and one buffer. These results will be utilized later in this paper.

Consider a fluid queue driven by the process $X(t)$, where $X(t)$ is now taken to be the number of customers present in an $M / G / 1 / 1 / 1$ queue. In other words, we make the assumption that there is a single on-off source, with exponentially distributed silent periods and generally distributed active periods. Let $S_{0}$ and $A_{0}$ denote a typical silent 
period and a typical active period of the source, respectively. We denote $\lambda=1 / E\left[S_{0}\right]$, $\beta(\theta)=E\left[e^{-\theta A_{0}}\right]$, and $\beta_{k}=E\left[A_{0}^{k}\right]$. Following the notation of Section 2, we denote the output rate of the source by $c_{0}$ and the leak rate of the buffer by $c_{1}$. Since there is only one source, we may assume that

$$
c_{0}>c_{1}
$$

\subsection{Output from the buffer}

Under Assumption (3.1) the output from the buffer looks like another on-off source: when non-empty, the buffer is leaking out with rate $c_{1}$, and when empty, no fluid flows out. Thus, the empty periods (non-empty periods) of the buffer are the same as the silent periods (active periods) of the output rate process. This fluid system is stable if and only if $c_{0} \beta_{1}<c_{1}\left(\beta_{1}+1 / \lambda\right)$, or equivalently, if

$$
\rho \equiv \frac{c_{0}}{c_{1}} \frac{\lambda \beta_{1}}{1+\lambda \beta_{1}}<1
$$

Let $S_{1}$ and $A_{1}$ denote a typical empty period and a typical non-empty period of the buffer, respectively. It is easy to see that empty periods are independently and exponentially distributed with mean

$$
E\left[S_{1}\right]=1 / \lambda .
$$

The non-empty periods are characterized by the following lemma. For the proof, see e.g. Proposition 1 in [2].

Lemma 3.1. Non-empty periods $A_{1}$ are distributed as busy periods in an $M / G / 1$ queue with arrival rate $\lambda\left(c_{0}-c_{1}\right) / c_{0}$ and Laplace-Stieltjes transform of the service time distribution given by $\beta\left(\theta c_{0} / c_{1}\right)$. In addition, this $M / G / 1$ queue is stable if and only if $\rho<1$.

\subsection{Content of the buffer}

We now turn to the stationary distribution of the content of the buffer. Let $Z$ denote the (stationary) buffer content. First we note that, under the assumption that $\rho<1$, the buffer content process is regenerative with cycles consisting of a non-empty period and the following empty period. It follows immediately that

$$
P\{Z>0\}=\frac{E\left[A_{1}\right]}{E\left[A_{1}\right]+E\left[S_{1}\right]}=\rho .
$$

The following lemma is due to Corollary 3 in Kella and Whitt [8].

Lemma 3.2. Let $V$ denote the stationary version of the workload in an $M / G / 1$ queue with arrival rate $\lambda / c_{1}$ and Laplace-Stieltjes transform of the service time distribution given by $\beta\left(\left(c_{0}-c_{1}\right) \theta\right)$. This queue is stable if and only if $\rho<1$. In the stable case, for all $z \geq 0$,

$$
P\{Z>z\}=\gamma P\{V>z\}
$$

where

$$
\gamma \equiv \frac{P\{Z>0\}}{P\{V>0\}}=\frac{c_{0}}{c_{0}-c_{1}} \frac{1}{1+\lambda \beta_{1}},
$$


Corollary 3.3. If $\rho<1$, then

$$
E\left[e^{-\theta Z}\right]=1-\gamma+\gamma \frac{\left(c_{1}-\left(c_{0}-c_{1}\right) \lambda \beta_{1}\right) \theta}{c_{1} \theta-\lambda+\lambda \beta\left(\left(c_{0}-c_{1}\right) \theta\right)}
$$

Proof. From Lemma 3.2 it follows that $E\left[e^{-\theta Z}\right]=1-\gamma+\gamma E\left[e^{-\theta V}\right]$. Then apply the Pollaczek-Khintchine formula for $E\left[e^{-\theta V}\right]$.

Corollary 3.4. If $\rho<1$, then

$$
\begin{aligned}
& E[Z]=\frac{\beta_{2}}{2 \beta_{1}} \frac{c_{0}-c_{1}}{1+\lambda \beta_{1}} \frac{\rho}{1-\rho}, \\
& E\left[Z^{2}\right]=\frac{\beta_{3}}{3 \beta_{1}} \frac{\left(c_{0}-c_{1}\right)^{2}}{1+\lambda \beta_{1}} \frac{\rho}{1-\rho}+2\left(\frac{\beta_{2}}{2 \beta_{1}}\right)^{2} \frac{\left(c_{0}-c_{1}\right)^{3}}{c_{0}\left(1+\lambda \beta_{1}\right)}\left(\frac{\rho}{1-\rho}\right)^{2} .
\end{aligned}
$$

Proof. From Lemma 3.2 it follows that $E\left[Z^{k}\right]=\gamma E\left[V^{k}\right]$ for all $k \geq 1$. Then apply the Pollaczek-Khintchine formulas for the first moments of $V$.

\section{Main Results: MUltiple SOURCES, MUltiple BufFers}

In this section we present new results concerning a tandem fluid queue fed by multiple on-off sources. First we characterize the output from each of the buffers (Theorem 4.1). Then we derive an implicit formula for the Laplace-Stieltjes transform (Theorem 4.4) and explicit expressions for the first moments (Theorem 4.5) of the marginal content distribution of any buffer $j \geq 2$. The Laplace-Stieltjes transform is found explicitly and inverted for the case in which the active periods of the sources are exponentially distributed (Theorem 4.6). Furthermore, we obtain an explicit expression for the correlation coefficient between the contents of any two buffers $i, j \geq 2$ (Theorem 4.7). The results are formulated for a finite number of sources. In Subsection 4.5 we explain how they extend to the case $N=\infty$. If there is only one source, all the results also hold for the first buffer $(j=1)$.

We take the process $X(t)$ that modulates the tandem fluid queue now to be the number of customers in an $M / G / N / N / N$ queue $(N<\infty)$. So, there is a series of fluid buffers, the first of which is fed by $N$ independent homogeneous on-off sources with exponential silent periods and generally distributed active periods. The notation used to describe these sources is the same as in Section 3. For the notation used to describe the tandem system, we refer to Section 2. In addition, we denote, for all $k$,

$$
\tilde{\beta}_{k} \equiv E\left[\left(c_{0} A_{0}\right)^{k}\right]=\beta_{k} c_{0}^{k} \text {. }
$$

Note that $c_{0} A_{0}$ is the amount of fluid generated in an active period $A_{0}$ of a source. As in Section 2, we make the assumptions (2.1) and (2.2) concerning the buffer rates. Furthermore, we define, for all $j \geq 1$,

$$
\rho_{j}=\frac{\bar{c}}{c_{j}} \text { and } \kappa_{j}=\frac{\bar{c}}{c_{j}-\bar{c}}
$$

where $\bar{c}$ is the mean input rate to the first buffer,

$$
\bar{c}=N c_{0} \frac{\lambda \beta_{1}}{1+\lambda \beta_{1}} \text {. }
$$


Notice that $\kappa_{j}$ can also be given as

$$
\kappa_{j}=\frac{\rho_{j}}{1-\rho_{j}}, \quad j \geq 1
$$

In addition, we let

$$
\kappa_{0}=\frac{\bar{c}}{N c_{0}-\bar{c}}
$$

Notice that, if $N=1$, then $\kappa_{0}=\lambda \beta_{1}$.

\subsection{Output from the $j$ th buffer}

As mentioned in Section 2, under assumptions (2.1) and (2.2), the output from any buffer $j \geq 1$ looks like an on-off source: the empty periods (non-empty periods) of the buffer are the same as the silent periods (active periods) of the corresponding output rate process. Since the first buffer can be empty only if all the $N$ sources are silent, and buffer $j+1$ can be empty only if buffer $j$ is empty (by Proposition 2.1), we deduce that, for any $j \geq 1$, the empty periods $S_{j}$ of buffer $j$ are independently and exponentially distributed with mean

$$
E\left[S_{j}\right]=1 /(N \lambda)
$$

The non-empty periods $A_{j}$ are characterized in the following theorem.

Theorem 4.1. Let $j \geq 1$. Non-empty periods $A_{j}$ are distributed as busy periods in an $M / G / 1$ queue with arrival rate $\lambda\left(N c_{0}-c_{j}\right) / c_{0}$ and Laplace-Stieltjes transform of the service time distribution given by $\beta\left(\theta c_{0} / c_{j}\right)$. In addition, this $M / G / 1$ queue is stable if and only if $\rho_{j}<1$.

Proof. For $j=1$, the theorem is proved in [5, 2]. However, due to Proposition 2.2 (take $i=1$ ), the result immediately generalizes to any $j \geq 1$.

We get the following corollaries for the Laplace-Stieltjes transform $\alpha_{j}(\theta)=E\left[e^{-\theta A_{j}}\right]$ and the first moments of $A_{j}$, which will be needed for Theorem 4.5 .

Corollary 4.2. Let $j \geq 1$. If $\rho_{j}<1$, then $\alpha_{j}(\theta)$ is the unique solution, with the property $\left|\alpha_{j}(\theta)\right| \leq 1$, of the following implicit equation:

$$
x=\beta\left(\frac{c_{0}}{c_{j}}\left[\theta+\lambda\left(N-\frac{c_{j}}{c_{0}}\right)(1-x)\right]\right), \quad \operatorname{Re} \theta \geq 0 .
$$

Corollary 4.3. Let $j \geq 1$. If $\rho_{j}<1$, then

$$
\begin{aligned}
& E\left[A_{j}\right]=\frac{\kappa_{j}}{N \lambda} \\
& E\left[A_{j}^{2}\right]=\beta_{2} \frac{c_{j}}{c_{0}}\left(\frac{\kappa_{j}}{N \lambda \beta_{1}}\right)^{3}, \\
& E\left[A_{j}^{3}\right]=\beta_{3} \frac{c_{j}}{c_{0}}\left(\frac{\kappa_{j}}{N \lambda \beta_{1}}\right)^{4}+3 \lambda \beta_{2}^{2} \frac{c_{j}}{c_{0}}\left(N-\frac{c_{j}}{c_{0}}\right)\left(\frac{\kappa_{j}}{N \lambda \beta_{1}}\right)^{5} .
\end{aligned}
$$




\subsection{Content of the $j$ th buffer}

Now we consider the (stationary) buffer content $Z_{j}$. We note that results concerning the first buffer $(j=1)$ have only been derived for $N=1$, see Section 3 (unless the on-times of the sources are expontentially distributed, see [3]). Therefore, we assume in this subsection that $j \geq 2$, or that $j=1$ and $N=1$.

By Theorem 4.1, the input to buffer $j$ looks like an on-off source with exponential silent periods and generally distributed active periods. This implies that we may apply the results of Section 3 to get formulas for the Laplace-Stieltjes transform and the first moments of $Z_{j}$. All we have to do, is replace $c_{0}, c_{1}, S_{0}, S_{1}, A_{0}$ and $A_{1}$ (and hence $\lambda, \beta(\cdot)$ and $\beta_{k}$ ) by $c_{j-1}, c_{j}, S_{j-1}, S_{j}, A_{j-1}$, and $A_{j}$ (and $N \lambda, \alpha_{j-1}(\cdot)$ and $E\left[A_{j-1}^{k}\right]$ ), respectively.

First, the stability condition given in Lemma 3.2 translates into

$$
\frac{c_{j-1}}{c_{j}} \frac{N \lambda E\left[A_{j-1}\right]}{1+N \lambda E\left[A_{j-1}\right]}<1
$$

which, using Corollary 4.3 and the fact that $\rho_{j-1} c_{j-1}=\rho_{j} c_{j}$, is easily seen to be equivalent to $\rho_{j}<1$. Note that the interpretation of $\rho_{j}$ is given by

$$
P\left\{Z_{j}>0\right\}=\frac{E\left[A_{j}\right]}{E\left[A_{j}\right]+E\left[S_{j}\right]}=\rho_{j} .
$$

Theorem 4.4. Let $j \geq 2$, or let $j=1$ and $N=1$. If $\rho_{j}<1$, then

$$
E\left[e^{-\theta Z_{j}}\right]=1-\gamma_{j}+\gamma_{j} \frac{\left(c_{j}-\left(c_{j-1}-c_{j}\right) \kappa_{j-1}\right) \theta}{c_{j} \theta-N \lambda+N \lambda \alpha_{j-1}\left(\left(c_{j-1}-c_{j}\right) \theta\right)},
$$

where $\alpha_{j-1}$ is given in Corollary 4.2 for $j \geq 2, \alpha_{0}(\cdot)=\beta(\cdot)$, and

$$
\gamma_{j} \equiv \frac{P\left\{Z_{j}>0\right\}}{P\left\{V_{j}>0\right\}}=\frac{c_{j-1}}{c_{j-1}-c_{j}}\left(1-\rho_{j-1}\right) .
$$

Proof. In both cases, the result is a consequence of Corollary 3.3. For $j=1$ and $N=1$, it is immediate, while for $j \geq 2$ we apply the substitutions as mentioned.

Theorem 4.5. Let $j \geq 2$, or let $j=1$ and $N=1$. If $\rho_{j}<1$, then

$$
\begin{aligned}
& E\left[Z_{j}\right]=\frac{\tilde{\beta}_{2}}{2 \tilde{\beta}_{1}}\left(\frac{1}{1+\lambda \beta_{1}}\right)^{2}\left(\kappa_{j}-\kappa_{j-1}\right), \\
& E\left[Z_{j}^{2}\right]=\frac{\tilde{\beta}_{3}}{3 \tilde{\beta}_{1}}\left(\frac{1}{1+\lambda \beta_{1}}\right)^{3} \frac{\left(\kappa_{j}-\kappa_{j-1}\right)^{2}}{\kappa_{j}}+ \\
& 2\left(\frac{\tilde{\beta}_{2}}{2 \tilde{\beta}_{1}}\right)^{2}\left(\frac{1}{1+\lambda \beta_{1}}\right)^{4} \frac{\left(\kappa_{j}-\kappa_{j-1}\right)^{2}\left(\kappa_{j-1}+\kappa_{j}-2 \lambda \beta_{1}\right)}{\kappa_{j}} .
\end{aligned}
$$

Proof. For $j=1$ and $N=1$, the result can easily be verified using Corollary 3.4. Now assume $j \geq 2$. We invoke Corollary 3.4 and substitute the moments of $A_{j-1}$ using Corollary 4.3. After strenuous rewriting and using (4.1) we find the claimed results.

We notice that, apart from the moments $\tilde{\beta}_{k}$, these expressions depend on the active period distribution only through the mean. 


\subsection{Content of the $j$ th buffer when the active periods are exponential}

When we assume that not only the silent periods but also the active periods of the sources are distributed according to an exponential distribution, with intensity $\mu$ say $\left(=1 / \beta_{1}\right)$, it is possible to invert the transform in Theorem 4.4.

Theorem 4.6. Let $j \geq 2$, or let $j=1$ and $N=1$. Furthermore, let the active periods of the sources be exponentially distributed with intensity $\mu$. If $\rho_{j}<1$, then

$$
\begin{aligned}
& P\left\{Z_{j} \in(y, y+d y)\right\}=\left(1-\rho_{j}\right) \delta_{0}(y) d y+\left(1-\rho_{j}\right) e^{-\eta_{j} y} \times \\
& \quad\left(\frac{c_{j-1}}{c_{j}} \frac{N \lambda}{c_{j-1}-c_{j}}-\frac{1}{2} \frac{N c_{0} \omega_{j}}{N c_{0}-c_{j}} \int_{0}^{y} e^{-\left(\Theta_{j}-\eta_{j}\right) u} \frac{I_{1}(u \sqrt{\omega})}{u \sqrt{\omega}} d u\right) d y,
\end{aligned}
$$

where $\delta_{0}$ denotes the Dirac measure at 0 and $I_{1}$ the modified Bessel function of the first kind of order 1. Furthermore, $\rho_{j}$ is defined as in (4.2) and the other parameters are given by

$$
\begin{aligned}
\Theta_{j} & =\frac{N \lambda+(\mu-\lambda) c_{j-1} / c_{0}}{c_{j-1}-c_{j}}, \\
\eta_{j} & =\frac{N \mu}{N c_{0}-c_{j}}-\frac{N \lambda}{c_{j}}, \\
\omega_{j} & =\frac{4 \lambda \mu c_{j-1}\left(N c_{0}-c_{j-1}\right)}{c_{0}^{2}\left(c_{j-1}-c_{j}\right)^{2}} .
\end{aligned}
$$

Proof. Since $A_{j-1}$ is now distributed as the busy period of an $M / M / 1$ system, its Laplace Stieltjes transform $\alpha_{j-1}(\theta)$ can be found explicitly. After putting things together and rewriting, we obtain

$$
\begin{aligned}
& E\left[e^{-\left(\theta-\Theta_{j}\right) Z_{j}}\right]=1-\rho_{j}+\left(1-\rho_{j}\right) \times \\
& \quad\left(\frac{c_{j-1}}{c_{j}} \frac{N \lambda}{c_{j-1}-c_{j}} \frac{1}{\theta-\left(\Theta_{j}-\eta_{j}\right)}-\frac{1}{2} \frac{N c_{0}}{N c_{0}-c_{j}} \frac{\theta-\sqrt{\theta^{2}-\omega_{j}}}{\theta-\left(\Theta_{j}-\eta_{j}\right)}\right) .
\end{aligned}
$$

Inversion can be done by using (28) on page 235 in [7].

We mention that the density of $Z_{1}$ with $N=1$ is exponential, since $\omega_{1}=0$ in this case.

\subsection{Correlation between buffer contents}

Returning to the case where the active periods of the sources are generally distributed, we now consider the correlation between the buffer contents $Z_{i}$ and $Z_{j}$ for some $j>i$. As before, we exclude the first buffer from this analysis in the general case, unless $N=1$. Throughout this subsection, we assume that $\beta_{k}<\infty$ for $k=1,2,3$.

Theorem 4.7. Let $j>i \geq 2$, or let $j>i=1$ and $N=1$. If $\rho_{j}<1$, then

$$
\operatorname{Corr}\left[Z_{i}, Z_{j}\right]=\frac{\sqrt{\kappa_{i}}}{\kappa_{j-1} \sqrt{\kappa_{j}}} \frac{b\left(\kappa_{i-1}+\kappa_{i}\right)+\kappa_{i-1}^{2}+\kappa_{i-1} \kappa_{i}+\kappa_{i}^{2}}{\sqrt{\left(2 b+2 \kappa_{i-1}+\kappa_{i}\right)\left(2 b+2 \kappa_{j-1}+\kappa_{j}\right)}}
$$

where the constant $b$ is defined as follows:

$$
b=\frac{2 \beta_{3} \beta_{1}}{3 \beta_{2}^{2}}\left(1+\lambda \beta_{1}\right)-2 \lambda \beta_{1} .
$$


Proof. By definition,

$$
\operatorname{Corr}\left[Z_{i}, Z_{j}\right]=\frac{\operatorname{Cov}\left[Z_{i}, Z_{j}\right]}{\sqrt{\operatorname{Var}\left[Z_{i}\right] \operatorname{Var}\left[Z_{j}\right]}}
$$

where $\operatorname{Cov}[\cdot, \cdot]$ and $\operatorname{Var}[\cdot]$ refer to covariance and variance, respectively. The variances $\operatorname{Var}\left[Z_{i}\right]$ and $\operatorname{Var}\left[Z_{j}\right]$ can be derived from the formulas presented in Theorem 4.5. As before, we let $Z_{i j}=Z_{i}+\ldots+Z_{j}$, with the convention that $Z_{i j}=0$ if $j<i$. Since

$$
2 Z_{i} Z_{j}=Z_{i j}^{2}+Z_{i+1, j-1}^{2}-Z_{i, j-1}^{2}-Z_{i+1, j}^{2}
$$

we obtain

$$
2 \operatorname{Cov}\left[Z_{i}, Z_{j}\right]=\operatorname{Var}\left[Z_{i j}\right]+\operatorname{Var}\left[Z_{i+1, j-1}\right]-\operatorname{Var}\left[Z_{i, j-1}\right]-\operatorname{Var}\left[Z_{i+1, j}\right] .
$$

By Proposition 2.2, all these variances can also be derived from the formulas presented in Theorem 4.5. After some straightforward manipulations the claimed result can be obtained.

Proposition 4.8. Let $j>i \geq 2$, or let $j>i=1$ and $N=1$. If $\rho_{j}<1$, then

$$
\operatorname{Corr}\left[Z_{i}, Z_{j}\right]>0 \text {. }
$$

Proof. In this proof we make use of the fact that

$$
\kappa_{M}>\kappa_{M-1}>\ldots>\kappa_{1}>0 .
$$

In fact, by $(2.1)$ we can show that $\kappa_{i}>\lambda \beta_{1}$ for any $i \geq 1$. By further taking into account that $\beta_{3} \beta_{1} \geq \beta_{2}^{2}$ for any active period distribution, we get the following lower bound for $b$ :

$$
b=\frac{2 \beta_{3} \beta_{1}}{3 \beta_{2}^{2}}\left(1+\lambda \beta_{1}\right)-2 \lambda \beta_{1} \geq \frac{2}{3}-\frac{4}{3} \kappa_{i-1}, \quad i=1, \ldots, M .
$$

It now follows that

$$
\begin{aligned}
b\left(\kappa_{i-1}\right. & \left.+\kappa_{i}\right)+\kappa_{i-1}^{2}+\kappa_{i-1} \kappa_{i}+\kappa_{i}^{2} \\
& \geq \frac{2}{3}\left(\kappa_{i-1}+\kappa_{i}\right)-\frac{4}{3} \kappa_{i-1}\left(\kappa_{i-1}+\kappa_{i}\right)+\kappa_{i}^{2}+\kappa_{i-1}\left(\kappa_{i-1}+\kappa_{i}\right) \\
& \geq \frac{2}{3}\left(\kappa_{i-1}+\kappa_{i}\right)-\frac{2}{3} \kappa_{i}^{2}+\kappa_{i}^{2} \geq 0,
\end{aligned}
$$

so that $\operatorname{Corr}\left[Z_{i}, Z_{j}\right]$ clearly is positive as well.

Consider now two consecutive buffers $i$ and $i+1(i \geq 2$, or $i \geq 1$ and $N=1)$. When the source characteristics are fixed, the correlation coefficient $\operatorname{Corr}\left[Z_{i}, Z_{i+1}\right]$ depends just on the rates $c_{i-1}, c_{i}$ and $c_{i+1}$. We now fix $c_{i-1}$ and $c_{i}$ for the time being (such that $c_{i-1}>c_{i}$ ), and vary $c_{i+1}$ between $\vec{c}$ and $c_{i}$. It can be shown that

(i) $\operatorname{Corr}\left[Z_{i}, Z_{i+1}\right] \rightarrow 0$ whenever $c_{i+1} \rightarrow \bar{c}$, and

(ii) $\operatorname{Corr}\left[Z_{i}, Z_{i+1}\right]$ is increasing and continuous as a function of $c_{i+1}$.

Let then $f\left(c_{i}\right)$ denote the limit of $\operatorname{Corr}\left[Z_{i}, Z_{i+1}\right]$ as $c_{i+1} \rightarrow c_{i}$. It can be shown that $f\left(c_{i}\right)$ is continuous on the interval $\left(\bar{c}, c_{i-1}\right)$, with $f(\bar{c})=1 / \sqrt{3}$ and $f\left(c_{i-1}\right)=1$, while in between $f\left(c_{i}\right) \geq 1 / 2$. It follows that, with $c_{i-1}$ fixed, the correlation coefficient takes all values from 0 to 1 , as $c_{i}$ and $c_{i+1}$ vary in the range $c_{i-1}>c_{i}>c_{i+1}>\bar{c}$. In addition, we find that, in any neighbourhood of the point $\left(c_{i}, c_{i+1}\right)=(\bar{c}, \bar{c})$, the correlation coefficient takes all values from 0 to $1 / \sqrt{3}$. 
Numerical example

As a numerical example we consider a tandem fluid model with one source and two buffers. Silent and active periods of the source are exponentially distributed with means $1 / \lambda=1 / 4$ and $\beta_{1}=1$. The source rate and the mean input rate are $c_{0}=1$ and $\bar{c}=1 / 5$, respectively. Thus, we have the following constraints for the leak rates of the two buffers: $1>c_{1}>c_{2}>1 / 5$. The correlation coefficient between the buffer contents, $\operatorname{Corr}\left[Z_{1}, Z_{2}\right]$, is plotted as a function of the rates $c_{1}$ and $c_{2}$ in Fig. 4.1. A warning might be in place here, since it is not clear from this figure that the correlation coefficient takes all values from 0 to $1 / \sqrt{3}$ in any neighbourhood of the point $\left(c_{1}, c_{2}\right)=(\bar{c}, \bar{c})$. In Fig. 4.2, the function $f\left(c_{1}\right)$, that is the limit of $\operatorname{Corr}\left[Z_{i}, Z_{i+1}\right]$ as $c_{2} \rightarrow c_{1}$, is plotted for this particular example.

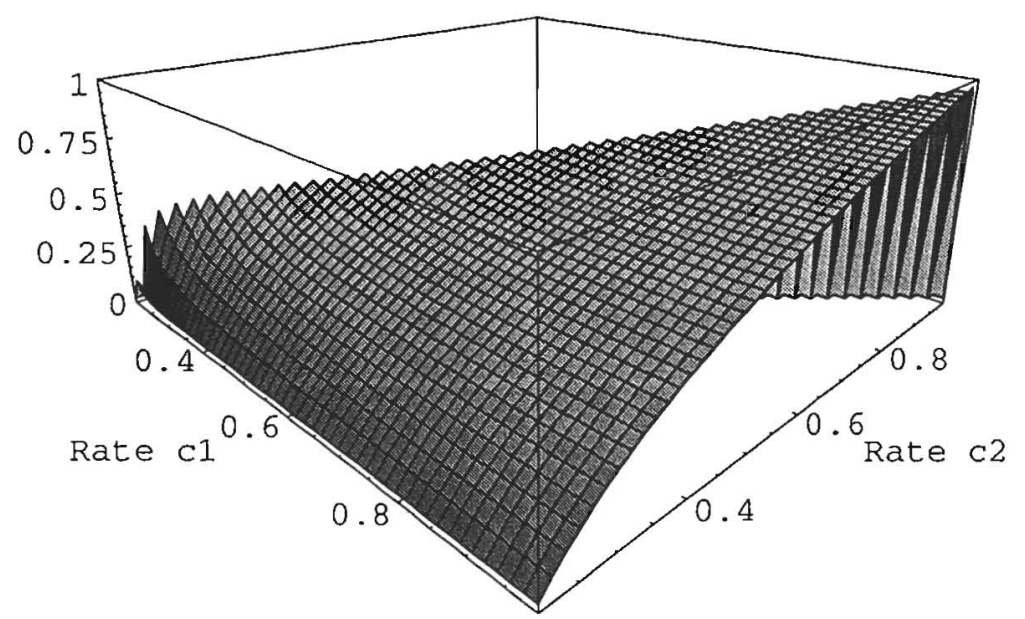

Frgure 4.1. Corr $\left[Z_{1}, Z_{2}\right]$ as a function of the rates $c_{1}$ and $c_{2}$.

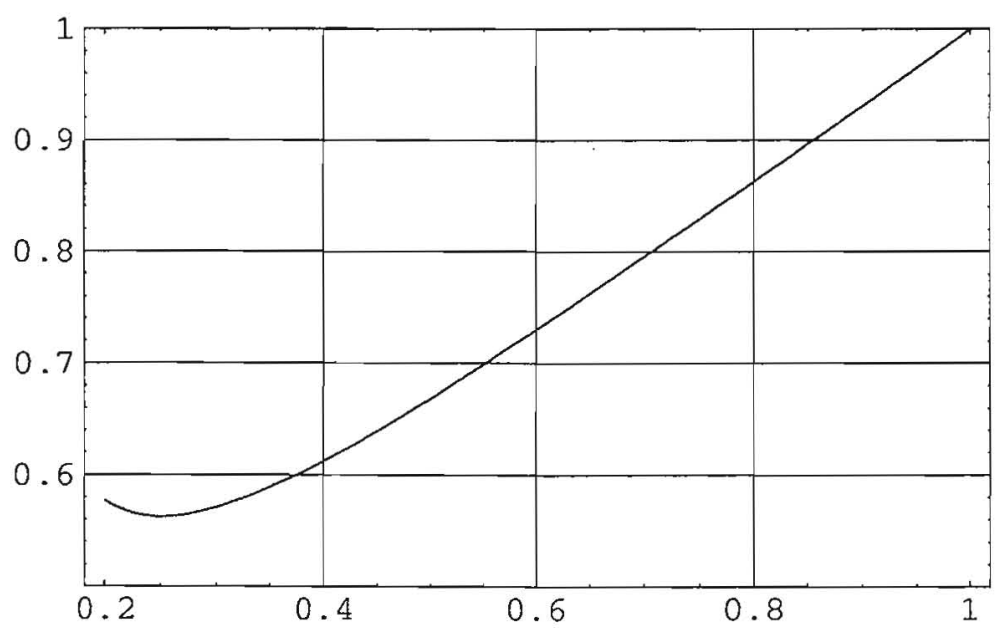

Figure 4.2. The limit $f\left(c_{1}\right)$ of $\operatorname{Corr}\left[Z_{1}, Z_{2}\right]$ as a function of the rate $c_{1}$. 


\subsection{Infinite number of sources}

Consider then the tandem fluid queue driven by the $M / G / \infty$ queue $(N=\infty)$. So, there is a series of fluid buffers, the first of which is fed by random bursts arriving according to a Poisson process with intensity $\nu$. Note that bursts in this model play the same role as the active periods in the previous model with finitely many sources. Clearly, the present model can be seen as a limiting case of the previous one: let $N \rightarrow \infty$ and $\lambda \rightarrow 0$ in such a way that $N \lambda \rightarrow \nu$.

The central observation here is that, under assumptions (2.1) and (2.2), the output from each buffer has a similar characterization as before: the empty periods $S_{j}$ of buffer $j$ are independently and exponentially distributed with mean $E\left[S_{j}\right]=1 / \nu$, and the non-empty periods $A_{j}$ are distributed as busy periods in an $M / G / 1$ queue with arrival rate $\nu$ and Laplace-Stieltjes transform of the service time distribution given by $\beta\left(\theta c_{0} / c_{j}\right)$. For $j=1$, these results were proved in [5]. However, again due to Proposition 2.2 (take $i=1$ ), the result immediately generalizes to any $j \geq 1$.

It follows that all the other results derived earlier in this section have their counterparts in the present model: just replace $\lambda$ by $\nu / N$ and then let $N \rightarrow \infty$.

\subsection{Tandem fluid model presented in [9]}

As mentioned in Section 1, the tandem fluid model presented in [9] can be seen as a limiting case of our model with a single source $(N=1)$ : the rate $c_{0}$ tends to $\infty$ and the active periods tend to 0 . It follows that, for example, the correlation between the contents of any two buffers $i$ and $j$ (in the model of [9]) has the same formula (4.12) as in our model but with $b=2 \tilde{\beta}_{3} \tilde{\beta}_{1} /\left(3 \tilde{\beta}_{2}^{2}\right)$. The $\kappa$ 's are defined as in (4.2) with $\bar{c}=\lambda \tilde{\beta}_{1}$ (and $\kappa_{0}=0$ ). As mentioned in [9], the correlation between the first two buffers is always in the interval $(0,1 / \sqrt{3})$. However, the correlation between two consecutive buffers from the second buffer on can have any value from 0 to 1 .

\section{ACKNOWLEDGEMENTS}

This work was performed during the first author's half-year visit to EURANDOM, Eindhoven. The visit was supported by the Academy of Finland. The authors wish to thank Prof. Onno Boxma for the fruitful discussions and comments on the present work.

\section{REFERENCES}

[1] S. Aalto, "Characterization of the output rate process for a Markovian storage model," J. Appl. Probab., vol. 35, pp. 184-199, 1998.

[2] S. Aalto, "Output of a multiplexer loaded by heterogeneous on-off sources," Commun. Stat., Stochastic Models, vol. 14, pp. 993-1005, 1998.

[3] D. Anick, D. Mitra, and M.M. Sondhi, "Stochastic theory of a data-handling system with multiple sources," Bell System Tech. J. vol. 61, 1871-1894, 1982.

[4] O.J. Boxma and V. Dumas, "Fluid queues with long-tailed activity period distributions," Computer-Communications, vol. 21, pp. 1509-1529, 1998.

[5] O.J. Boxma and V. Dumas, "The busy period in the fluid queue," Perf. Eval. Review, vol. 26, pp. 100-110, 1998.

[6] J.W. Cohen, "Superimposed renewal processes and storage with gradual input," Stochastic Processes Appl., vol. 2, pp. 31-58, 1974. 
[7] A. Erdelyi, Ed., Bateman Manuscript Project, Tables of Integral Transform, New York: Mc-Graw Hill, 1954.

[8] O. Kella and W. Whitt, "A storage model with a two-state random environment," Oper. Res. vol. 40, supp. 2, pp. S257-S262, 1992.

[9] O. Kella and W. Whitt, "A tandem fluid network with Lévy input," in Queues and Related Models, I. Basawa and U. Bhat, Eds., Oxford: Oxford University Press, 1992, pp. 112-128.

[10] O. Kella, "Parallel and tandem networks with dependent inputs," Ann. Appl. Probab. vol. 3, no. 3, pp. 682-695, 1993.

[11] O. Kella, "Stability and nonproduct form of stochastic fluid networks with Lévy inputs," Ann. Appl. Probab. vol. 6, no. 1, pp. 186-199, 1996.

[12] O. Kella, "Markov-modulated feedforward fluid networks," unpublished.

[13] I. Kino and M. Miyazawa, "The stationary work in system of a $G / G / 1$ gradual input model," J. Appl. Probab. vol. 30, pp. 207-222, 1993.

[14] L. Kosten, "Stochastic theory of a multi-entry buffer (I)," Delft Progress Report, vol. 1, pp. 10-18.

[15] D.P. Kroese and W.R.W. Scheinhardt, "Joint distributions for interacting fluid queues," unpublished.

[16] J.W. Roberts, U. Mocci, and J. Virtamo, Eds., Broadband Network Teletraffic - Final Report of Action COST 242, Berlin: Springer, 1996.

[17] M. Rubinovitch, "The output of a buffered data communication system," Stochastic Processes Appl., vol. 1, pp. 375-382, 1973.

[18] W.R.W. Scheinhardt, Markov-modulated and feedback fluid queues, Ph.D. Thesis, Faculty of Mathematical Sciences, University of Twente, Enschede, The Netherlands, 1998.

[19] K. Sigman and G. Yamazaki, "Fluid models with burst arrivals: a sample path analysis," Probab. Engrg. Inform. Sci., vol. 6, pp. 17-27, 1992.

(Samuli Aalto) Helsinki University of Technology, Laboratory of Telecommunications Technology, P.O. Box 3000, Fin-02015 HUT, Finland

E-mail address: samuli.aalto@hut.fi

(Werner Scheinhardt) Eindhoven University of Technology, Department of Mathematics and Computing Sciences, P.O. Box 513, 5600 MB Eindhoven, The Netherlands

E-mail address: werner@win.tue.nl 